\title{
Resonant Excitation of a Quantum Dot Strongly Coupled to a Photonic Crystal Nanocavity
}

\author{
Dirk Englund, ${ }^{1, *}$ Arka Majumdar, ${ }^{1}$ Andrei Faraon, ${ }^{2}$ Mitsuru Toishi, ${ }^{3}$ Nick Stoltz, ${ }^{4}$ Pierre Petroff, ${ }^{4}$ and Jelena Vučkovic ${ }^{1}$ \\ ${ }^{1}$ Department of Electrical Engineering, Stanford University, Stanford, California 94305, USA \\ ${ }^{2}$ Department of Applied Physics, Stanford University, Stanford, California 94305, USA \\ ${ }^{3}$ Sony Corporation, Shinagawa-ku, Tokyo, Japan, 141-0001 \\ ${ }^{4}$ Department of Electrical and Computer Engineering, University of California, Santa Barbara, California 93106, USA
}

(Received 13 August 2009; revised manuscript received 15 January 2010; published 19 February 2010)

We describe the resonant excitation of a single quantum dot that is strongly coupled to a photonic crystal nanocavity. The cavity represents a spectral window for resonantly probing the optical transitions of the quantum dot. We observe narrow absorption lines attributed to the single and biexcition quantum dot transitions and measure antibunched population of the detuned cavity mode $\left[g^{(2)}(0)=0.19\right]$.

DOI: 10.1103/PhysRevLett.104.073904

Photonic nanocavities coupled to semiconductor quantum dots (QDs) are becoming well developed systems for studying cavity quantum electrodynamics and constructing devices for quantum information processing. Recent experiments have found remarkably strong emission at the cavity resonance even when the QD emission was far detuned [1-4]. In these experiments the QD was pumped through the wetting layer, which allows for multiexciton processes through the wetting layer or a multi-QD effective continuum. In contrast, in this Letter we investigate the resonant excitation of an InAs quantum dot that is strongly coupled to a photonic crystal cavity. We show that the resonantly excited QD emits efficiently through the cavity mode and model the interaction by a phonon-mediated dephasing model [5-7]. In this experiment, the cavity signal effectively becomes a spectrally separated readout channel for high-resolution single quantum dot spectroscopy. In addition, because the QD is resonantly excited, the antibunched cavity emission has the potential for low timing jitter and high photon indistinguishability $[7,8]$.

The optical system consists of a photonic crystal (PC) cavity fabricated in a $160-\mathrm{nm}$ thick GaAs membrane which contains a central layer of self-assembled InGaAs QDs. As shown in Fig. 1(c), we employ a grating-integrated cavity (GIC) design [9], based on a linear three-hole defect cavity [10]. The GIC design incorporates a second-order grating around the cavity, as seen in the perturbations in Figs. 1(b) and 1(c), which couple plane waves with low in-plane $k$ vectors with the cavity mode.

We first characterize the QD-cavity system by its photoluminescence (PL) under nonresonant excitation at a temperature between 10 and $50 \mathrm{~K}$. A continuous-wave (cw) laser at $\lambda_{p}=860 \mathrm{~nm}$ excites electron-hole pairs which can relax through a phonon-mediated process into radiative levels of the QD [Fig. 1(d)]. Figure 1(e) plots the anticrossing between the QD-like and cavitylike states. Fits to the anticrossing spectra yield the system parameters summarized in Fig. 1(d).

We measure the reflection of the cavity by the crosspolarized reflectivity method illustrated in Fig. 1(a), as in
PACS numbers: 42.70.Qs, 42.50.Ct, 42.50.Dv, 78.67.Hc

our earlier work [11]. The cavity is polarized at $45^{\circ}$ to the vertical input beam, while the reflection is detected in the horizontal polarization on a charge coupled device (CCD). Figure 1(f) shows the vacuum Rabi splitting [11-13]. We
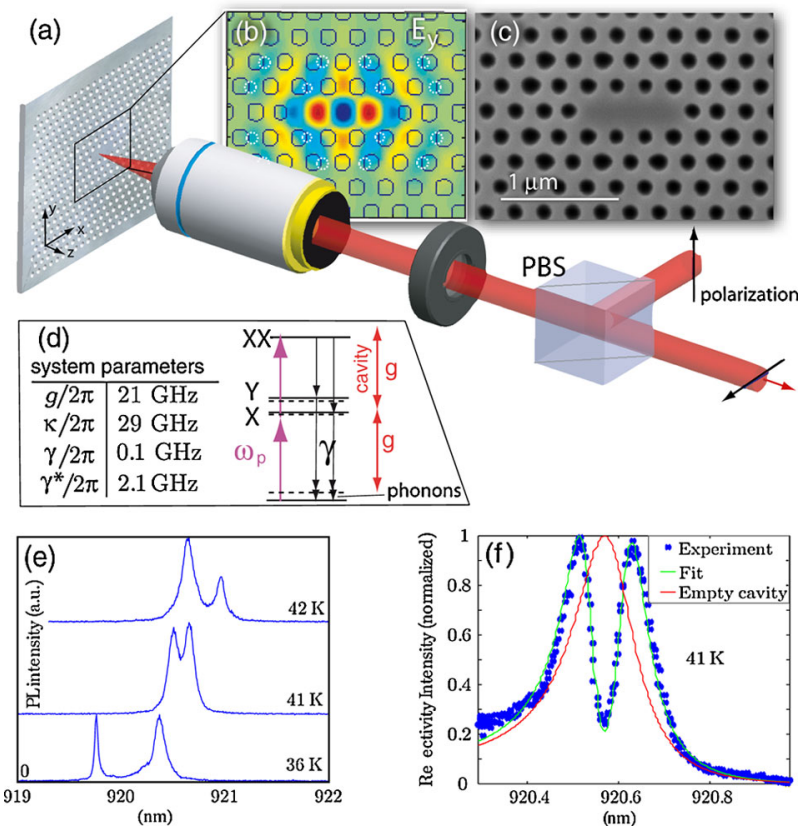

FIG. 1 (color online). (a) Cross-polarized confocal microscope setup. (b) $E_{y}$ field in the L3-cavity structure. The structure is perturbed at the sites indicated by dashed circles, as described in Ref. [9]. (c) Scanning electron micrograph of fabricated structure. (d) Energy levels of the coupled QD-cavity system showing the two polarization states of the single exciton $(X, Y)$ and the biexciton state $(X X)$. The table lists the system parameters derived from the measurements, where $g, \kappa, \gamma^{*}$ are the vacuum Rabi frequency, cavity field decay rate, and dipole dephasing rate, respectively. The dipole decay rate $\gamma$ is estimated from the lifetime of uncoupled QDs. (e) The photoluminescence (PL) shows the QD-cavity anticrossing as the QD is temperature tuned through the cavity. (f) Vacuum Rabi splitting observed in the reflectivity from the strongly coupled QD-cavity system. For comparison, we show the reflectivity of an empty cavity. 
obtain good agreement with a theoretical model, described later in the text [solid line fit in Fig. 1(f)], using the same parameters as derived from the photoluminescence.

When the QD exciton line is blue detuned from the cavity by $\delta=\lambda_{d}-\lambda_{c}=-1.17 \mathrm{~nm}$ at a temperature of $10 \mathrm{~K}$, the laser is scanned across the QD and cavity resonances, as shown in Fig. 2. The excitation laser power is $\sim 12 \mathrm{nW}$ before the objective lens. Precisely when the laser becomes resonant with the QD, we observe a strong emission into the cavity mode; otherwise, the cavity is dark. We can view the cavity as an efficient readout channel for resonant quantum dot spectroscopy: the QD emits into the cavity mode, which is far detuned and easy to separate spectrally. Alternatively, if the cavity mode is pumped, the QD single-exciton line radiates. Figures 2(b) and 2(c) plot spectra when the QD [cavity 2(c)] are pumped.

Figure 2(d) plots the integrated cavity emission as a function of the laser pump wavelength $\lambda_{p}$. We observe an absorption linewidth below $0.008 \mathrm{~nm}(2.7 \mathrm{GHz})$, which is better than $0.03 \mathrm{~nm}$, the resolution of our $75 \mathrm{~cm}$ spectrometer. The technique allows mapping of QD transitions with a resolution given by the excitation laser, which is in the $\mathrm{MHz}$ regime in our case. This cavity-enhanced spectroscopy technique adds an important tool to the repertoire for resonant single quantum dot spectroscopy [14-17]. Resonance fluorescence from a QD in a cavity was previously reported in a planar optical cavity [18]; however, the excitation geometry used in Ref. [18] is difficult to realize in cavity designs for high Purcell regime or strong
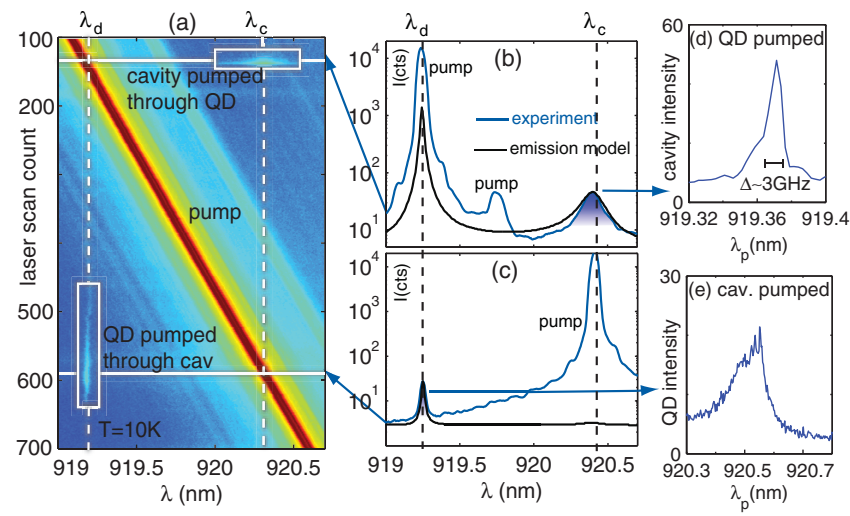

FIG. 2 (color). QD spectroscopy through cavity mode at $10 \mathrm{~K}$. (a) Intensity on spectrometer when the pump wavelength $\lambda_{p}$ is scanned across the detuned QD-cavity system (the laser has a weak red-detuned side band; power is $12 \mathrm{nW}$ before the objective lens). When $\lambda_{p}$ is resonant with the dot (cavity), the cavity (QD) intensity rises. (b) Spectrum when QD is pumped (the middle peak corresponds to the laser side mode) (c) spectrum when cavity is pumped. In (b) and (c), we model the driving mechanism by a pure dephasing process with $\gamma^{*}=0.1 g$. The model agrees well with the emission through the cavity (QD) when the QD (cavity) is pumped. (d) The integrated cavity emission as a function of the pump wavelength $\lambda_{p}$ shows the single-exciton absorption resolved to $3 \mathrm{GHz}$. (e) The integrated QD emission. coupling, such as photonic crystals or microdisks. The cavity-enhanced spectroscopy shown here should be universally applicable for solid state cavity QED systems with most cavity designs, as long as the QD has a large enough pure dephasing rate to drive the cavity.

Figure 2(e) plots the integrated QD intensity as the laser is scanned over the cavity resonance. The transfer to the QD is strongly asymmetric towards higher energy photons absorbed into the cavity, which are closer to the QD singleexciton wavelength.

The mechanism that allows the quantum dot to drive the far off-resonant cavity is described by a simple phonon dephasing model. It has previously been reported that quantum dots that were pumped through higher excited states or the QD wetting layer can drive the cavity even when it is far detuned [2-4]. Several recent theoretical models attribute the off-resonant driving of the cavity mode to a pure dephasing mechanism of the quantum dot [5-7]. We describe our experimental data with a pure dephasing rate $\gamma^{*}$ [19]. The dephasing term allows driving of the cavity (QD) through the QD (cavity), as is shown in the fit in Figs. 2(c) and 2(d), where we used $\gamma^{*}=0.1 \kappa$. This value of $\gamma^{*}$ was fit to match the off-resonant excitation between QD and cavity in Figs. 2(c) and 2(d) and was also estimated independently [see Fig. 4(c)] and agrees with values cited in the literature for resonant excitation studies [20]. The dephasing rate $\gamma^{*}=0.1 \kappa$ also explains the $2.7 \mathrm{GHz}$ linewidth of the exciton absorption [Fig. 2(d)], which is broader by a factor of 2 than the radiative linewidth corresponding to the exciton lifetime of $118 \mathrm{ps}$ at a similar QD-cavity detuning [Fig. 4(c)].

In Fig. 3(a), we explore the dependence of the cavity feeding mechanism on the QD single-exciton-cavity detuning $\delta$. We measured the cavity intensity through the resonantly driven QD for a temperature range of 28-47 K, corresponding to a QD-cavity detuning from -0.6 to $0.4 \mathrm{~nm}$. At each temperature given in Fig. 3(a), we tune the $\mathrm{cw}$-excitation laser to the QD, keeping the power constant at $50 \pm 0.5 \mathrm{nW}$ before the objective lens. In the
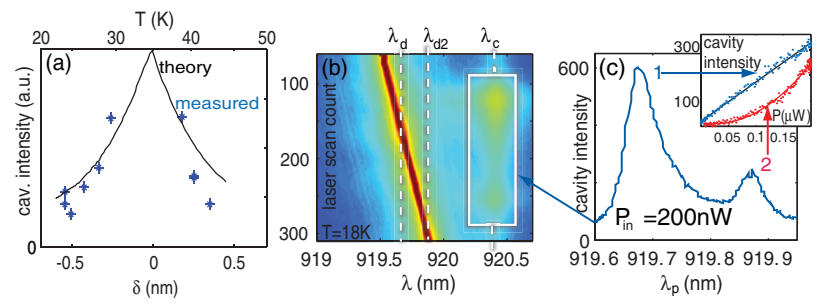

FIG. 3 (color). (a) Dependence of integrated cavity emission on QD detuning when the QD is resonantly pumped. (b) Pump power dependence: at a pump power of $200 \mathrm{nW}$, a second line becomes visible at a pump wavelength $\lambda_{p}=919.87 \mathrm{~nm}$. (The lines are slightly shifted since the temperature was, for technical reasons, raised to $18 \mathrm{~K}$ ). (c) Integrated cavity emission as $\lambda_{p}$ is scanned. Inset: The power dependence of the two lines suggests exciton $(X)$ and biexciton $(X X)$ states; in this inset, a background of 40 was subtracted. 
dephasing model, shown in the black curve in Fig. 3(a), we assume a temperature-dependent dephasing [21] rate $\gamma=\gamma_{0}+\alpha_{0} T$, with $\alpha_{0}=0.5 \mu \mathrm{eV} \mathrm{K}^{-1}$ and $\gamma_{0}=$ $\kappa / 100$. We use the dephasing model to describe the experimental data at small detunings on the order of $\mathrm{meV}$, where the dephasing processes are thought to be mediated by absorption or emission of acoustic phonons [21]. In the regime of larger detunings, up to tens of $\mathrm{meV}$, the efficient cavity pumping persists through a process that has recently been attributed to transitions between excited multiexciton states [22].

Figure 3(b) shows the resonant excitation repeated at a higher power of $P_{\text {in }}=200 \mathrm{nW}$. One striking difference is that the features are far more blurred; we believe this results from power broadening and increased spectral diffusion at high intensity [21,23]. A scan across the QD, where the tail of the excitation laser is subtracted, gives the cavity emission spectrum shown in Fig. 3(c). The data reveal a second peak when the pump is tuned to $\lambda_{p}=$ $919.85 \mathrm{~nm}$. In Fig. 3(c) (inset), we plot the cavity emission as a function of excitation power when $\lambda_{p}$ is tuned to two resonant wavelengths. The single-exciton line shows a linear pump dependence, as expected. The second line shows a quadratic pump dependence. The power dependence suggests a biexciton state that is resonantly pumped by two-photon absorption; we note, however, that the $X-X X$ energy splitting is rather low compared to values reported for resonant $X X$ excitation in bulk semiconductor [24]. Unfortunately, the signal-to-background was too low to confirm the biexciton nature of the second peak by crosscorrelation measurements with the single-exciton emission.

We now describe autocorrelation measurements under resonant excitation. At low temperature, the exciton-cavity detuning is large enough so that the single-exciton can be resonantly excited while very little pump light overlaps
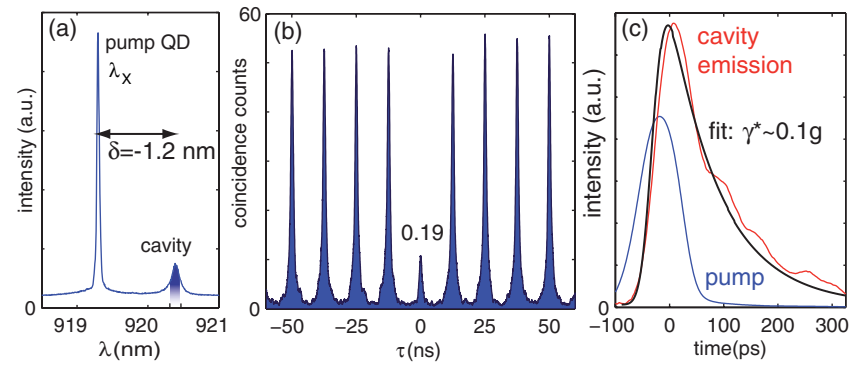

FIG. 4 (color online). (a) Resonant QD excitation at $10 \mathrm{~K}$. For the autocorrelation measurements, the cavity emission is isolated from the pump using a grating filter providing $17 \mathrm{~dB}$ of isolation at $\delta=-1.2 \mathrm{~nm}$ detuning. The laser is operated in continuous wave here so that is has low overlap with the cavity. (b) Autocorrelation histogram of cavity emission when the QD is pumped resonantly with 40-ps pulses (spectrum not shown). (c) Time-resolved resonant pumping of the quantum dot and emission into the cavity mode. A theoretical fit to the cavity emission yields an estimate of the pure dephasing rate. with the cavity resonance. This is shown in Fig. 4(a) for the narrow cw-wave pump laser. We spectrally filter the cavity emission using a dispersion grating-based filter (FWHM of $0.2 \mathrm{~nm}$ ) before sending the signal to a Hanbury BrownTwiss (HBT) setup. Under the cw-excitation configuration, we observe only weak antibunching $\left(g^{(2)}(0)=0.73(5)\right)$ because the features are blurred by the 300 ps detector response time, which is slower than the $\sim 118$ ps exciton lifetime (see below). To overcome the detector timing resolution, we excite the single-exciton line with $40 \mathrm{ps}$ pulses at a repetition rate of $80 \mathrm{MHz}$. The center excitation wavelength is resonant with the QD at $919.5 \mathrm{~nm}$. Figure 4(b) shows the autocorrelation histogram of the cavity emission, which indicates $g^{(2)}(0)=0.19(1)$. The clear antibunching is markedly different from previous reports which observed weak or no antibunching of the detuned PC cavity mode [2,3], and may be attributed to the expected absence of multiexciton population of the QD under resonant excitation of the single-exciton [25]. A significant contribution to the value of $g^{(2)}(0)$ is due to overlap of the 40-ps pulses with the filter at the cavity frequency, which could be improved by a narrower filter or larger QD-cavity detuning.

The autocorrelation measurements demonstrate the use of the resonantly driven QD as an on-demand single photon source. The resonant driving mechanism can reduce timing jitter due to the random relaxation time of electron-hole pairs under above-resonant excitation. The reduction in jitter would lead to an improvement in photon indistinguishability [7]. Furthermore, the emission wavelength is given by the cavity frequency and would be less sensitive to spectral jumps in the QD emission wavelength. A maximum wave packet overlap of $\sim 0.81$ has been reported for a QD coupled to a micropost [26], 0.72 for a photonic crystal cavity [27], and 0.67 for a waveguide-coupled cavity by this group [28]. In this experiment, the cavity emission under resonant excitation of the QD was too low to measure the indistinguishability, in part because of low coupling efficiency $(\sim 0.1)$ into the single mode fiber in our Hong-Ou-Mandel setup.

We performed time-resolved measurements of the emission of the resonantly excited QD into the cavity mode. Figure 4(c) shows the 40-ps pump pulse and cavity emission, measured simultaneously on a streak camera. The QD-driven cavity emission lifetime is $\tau \sim 118$ ps when the dot is detuned by $\delta=-1.2 \mathrm{~nm}$. We can use this lifetime measurement to infer the dephasing rate. We fit the decay time by a Monte Carlo simulation of the master equation, with the QD initialized in the ground state and resonantly excited by the 40-ps pump pulse [29]. All parameters except for $\gamma^{*}$ are fixed as before. The fit gives $\gamma^{*}=$ $0.10(1) g$.

In conclusion, we have studied the resonant excitation of a strongly coupled QD-cavity system, which shows a strong dependence on the off-resonant QD-cavity interaction. In contrast to previous experiments in which the QD 
was excited through higher excited states [2-4,30], the resonant driving of the QD reduces the probability of multiexciton population of the dot. We showed that the cavity represents a convenient readout channel for highresolution, resonant single quantum dot spectroscopy studies; this feature has recently also been reported for a QD-micropillar cavity system [31]. Since the cavity emission is strongly antibunched, it promises a single photon source with the potential for high photon indistinguishability [7]. In future studies, the collection efficiency from the cavity mode can be improved by employing integrated photonic crystal structures, where cavities coupled to single quantum dots may be connected through networks of waveguides [28,32-34] and other chip-integrated elements.

Financial support was provided by the ONR, NSF, ARO, and DARPA; A. M. was supported by the SGF. Work was performed in part at the Stanford Nanofabrication Facility of NNIN supported by the NSF. We thank Hideo Mabuchi for helpful discussions.

*Present address: Department of Physics, Harvard University, Cambridge MA 02138, USA. englund@fas.harvard.edu

[1] T. Yoshie, A. Scherer, J. Hendrickson, G. Khitrova, H. M. Gibbs, G. Rupper, C. Ell, O. B. Shchekin, and D. G. Deppe, Nature (London) 432, 200 (2004).

[2] K. Hennessy, A. Badolato, M. Winger, D. Gerace, M. Atature, S. Gulde, S. Falt, E. L. Hu, and A. Imamoglu, Nature (London) 445, 896 (2007).

[3] M. Kaniber, A. Laucht, A. Neumann, J. M. Villas-Bôas, M. Bichler, M.-C. Amann, and J. J. Finley, Phys. Rev. B 77, 161303(R) (2008).

[4] S. Strauf, K. Hennessy, M. T. Rakher, Y.-S. Choi, A. Badolato, L. C. Andreani, E. L. Hu, P. M. Petroff, and D. Bouwmeester, Phys. Rev. Lett. 96, 127404 (2006).

[5] A. Naesby, T. Suhr, P. T. Kristensen, and J. Mörk, Phys. Rev. A 78, 045802 (2008).

[6] Makoto Yamaguchi, Takashi Asano, and Susumu Noda, Opt. Express 16, 18067 (2008).

[7] Alexia Auffèves, Jean-Michel Gérard, and Jean-Philippe Poizat, Phys. Rev. A 79, 053838 (2009).

[8] A. Kiraz, M. Atatüre, and I. Imamoǧlu, Phys. Rev. A 69, 032305 (2004).

[9] M. Toishi, D. Englund, A. Faraon, and J. Vučković, Opt. Express 17, 14618 (2009).

[10] Y. Akahane, T. Asano, B.-S. Song, and S. Noda, Nature (London) 425, 944 (2003).

[11] D. Englund, A. Faraon, I. Fushman, N. Stoltz, P. Petroff, and J. Vučković, Nature (London) 450, 857 (2007).

[12] R. J. Thompson, G. Rempe, and H. J. Kimble, Phys. Rev. Lett. 68, 1132 (1992).

[13] E. Waks and J. Vučković, Phys. Rev. Lett. 96, 153601 (2006).
[14] M. Kroner, C. Lux, S. Seidl, A. W. Holleitner, K. Karrai, A. Badolato, P. M. Petroff, and R. J. Warburton, Appl. Phys. Lett. 92, 031108 (2008).

[15] Benito Alén, Florian Bickel, Khaled Karrai, Richard J. Warburton, and Pierre M. Petroff, Appl. Phys. Lett. 83, 2235 (2003)

[16] Alexander Högele, Stefan Seidl, Martin Kroner, Khaled Karrai, Richard J. Warburton, Brian D. Gerardot, and Pierre M. Petroff, Phys. Rev. Lett. 93, 217401 (2004).

[17] A. N. Vamivakas, M. Atature, J. Dreiser, S. T. Yilmaz, A. Badolato, A. K. Swan, B. B. Goldberg, A. Imamoglu, and M. S. Unlu, Nano Lett. 7, 2892 (2007).

[18] A. Muller, E. B. Flagg, P. Bianucci, X. Y. Wang, D. G. Deppe, W. Ma, J. Zhang, G. J. Salamo, M. Xiao, and C. K. Shih, Phys. Rev. Lett. 99, 187402 (2007).

[19] We found the Maxwell-Bloch equations for the coupled cavity-QD system using dephasing rate as a phenomenological parameter. Using the quantum regression theorem, we calculated the spectrum of the coupled cavity-QD system, as described in Ref. [7].

[20] W. Langbein, P. Borri, U. Woggon, V. Stavarache, D. Reuter, and A. D. Wieck, Phys. Rev. B 70, 033301 (2004).

[21] I. Favero, A. Berthelot, G. Cassabois, C. Voisin, C. Delalande, Ph. Roussignol, R. Ferreira, and J. M. Gérard, Phys. Rev. B 75, 073308 (2007).

[22] M. Winger et al., arXiv:0907.1881v1.

[23] A. Berthelot, I. Favero, G. Cassabois, C. Voisin, C. Delalande, Ph. Roussignol, R. Ferreira, and J.M. Gerard, Nature Phys. 2, 759 (2006).

[24] Gang Chen, T. H. Stievater, E. T. Batteh, Xiaoqin Li, D. G. Steel, D. Gammon, D. S. Katzer, D. Park, and L. J. Sham, Phys. Rev. Lett. 88, 117901 (2002).

[25] G. Tarel and V. Savona, Phys. Status Solidi C 6, 902 (2009).

[26] C. Santori, D. Fattal, J. Vučković, G. S. Solomon, and Y. Yamamoto, Nature (London) 419, 594 (2002).

[27] S. Laurent, S. Varoutsis, L. Le Gratiet, A. Lemaître, I. Sagnes, F. Raineri, A. Levenson, I. Robert-Philip, and I. Abram, Appl. Phys. Lett. 87, 163107 (2005).

[28] D. Englund, A. Faraon, B. Zhang, Y. Yamamoto, and J. Vuckovic, Opt. Express 15, 5550 (2007).

[29] The values for $g, \kappa, \gamma$, and $\Delta \lambda$ are taken from the spectral characterization. The Jaynes Cummings Hamiltonian includes the driving term $E(t)\left(\sigma_{+}+\sigma_{-}\right)$, where $\sigma_{+,-}$are the raising and lowering operators of the quantum dot, and the classical field $E(t)=E_{0} \exp \left(-t^{2} / 2 \sigma_{t}^{2}\right)$ has a FWHM of 40 ps.

[30] A. Laucht, N. Hauke, J. M. Villas-Boas, F. Hofbauer, G. Bohm, M. Kaniber, and J. J. Finley, Phys. Rev. Lett. 103, 087405 (2009).

[31] S. Ates, S. M. Ulrich, A. Ulhaq, S. Reitzenstein, A. Loffler, S. Hofling, A. Forchel, and P. Michler, Nat. Photon. 3, 724 (2009)

[32] A. Faraon, I. Fushman, D. Englund, N. Stoltz, P. Petroff, and J. Vuckovic, Opt. Express 16, 12154 (2008).

[33] H. Takano, B. S. Song, T. Asano, and S. Noda, Opt. Express 14, 3491 (2006).

[34] T. Tanabe, M. Notomi, E. Kuramochi, A. Shinya, and H. Taniyama, Nat. Photon. 1, 49 (2007). 